\title{
Secret médical en prison: le mythe de l'expert permanent
}

\section{Philippe Ducor}

Avocat, professeur à la Faculté de droit de l'Université de Genève, Médecin spécialiste en médecine interne

\section{Contexte}

Dans un espace de quelques mois en 2013, deux jeunes femmes - Marie et Adeline - ont été assassinées par des condamnés qui avaient bénéficié d'un allégement dans l'exécution de leur peine au sens de l'art. 75a al. 2 du code pénal $(\mathrm{CP})$. Suite à ces événements, la Conférence Latine des chefs des Départements de Justice et Police (CLDJP) a formulé une recommandation le 31 octobre 2013. Ce document recommande aux cantons d'adapter leurs législations de manière à ce que les médecins et autres thérapeutes soient «libérés» du secret médical et du secret de fonction à l'égard des autorités, dès lors qu'il s'agit de les informer sur des faits importants en matière de dangerosité. Les cantons de Genève, de Vaud et du Valais ont décidé de mettre en œuvre cette recommandation, en allant toutefois au-delà de ses exigences. En effet, leurs modifications législatives ne se contentent pas d'assouplir le secret médical, mais instituent pour les soignants une véritable obligation d'informer l'autorité.

\section{Système actuel}

La réglementation actuelle du secret médical découle des articles 321 et 17 du code pénal (CP). Selon l'article $321 \mathrm{ch} .1 \mathrm{CP}$, est punissable le médecin qui révèle un secret qui lui est confié en vertu de sa profession. Cette obligation de confidentialité n'est toutefois pas absolue. Selon l'article 321 ch. 2 CP, le médecin est délié du secret, c'est-à-dire autorisé à parler si le patient l'y autorise ou si, à la demande du médecin, l'autorité cantonale de levée du secret l'y autorise par écrit. L'art. 321 ch. $3 \mathrm{CP}$ réserve par ailleurs les dispositions de la législation fédérale ou cantonale prévoyant une obligation de renseigner ou de témoigner en justice. ${ }^{1}$ L'art. $17 \mathrm{CP}$ énonce quant à lui un motif justificatif, fondé sur l'imminence de l'atteinte à un bien juridique (état de nécessité licite). Lorsque le médecin viole le secret médical à l'égard d'un détenu afin de préserver un tel bien par exemple la vie ou l'intégrité corporelle de tiers -, sauvegardant ainsi un intérêt prépondérant, il agit de manière licite et ne commet aucune infraction.
Selon la réglementation actuelle, les médecins carcéraux sont ainsi autorisés - et non obligés - à passer outre le consentement du détenu et donc informer l'autorité, dans les cas suivants:

- lorsque ils ont été déliés du secret par l'autorité de levée du secret après l'avoir saisie. Cette voie n'est toutefois pas praticable lorsque le danger est imminent;

- d'emblée lorsqu'existe un état de nécessité licite au sens de l'article $17 \mathrm{CP}$, c'est-à-dire un danger imminent.

Or le médecin carcéral n'est ni formé, ni qualifié à l'évaluation de la dangerosité des détenus.

C'est ainsi que le médecin effectue lui-même la nécessaire pesée des intérêts qui le mène à saisir l'autorité de levée du secret ou à informer d'emblée les autorités. Cette solution est appropriée, dans la mesure où le médecin est directement engagé dans la relation thérapeutique et mieux placé que quiconque pour apprécier la situation. Dans la pratique, il arrive que le médecin carcéral saisisse l'autorité de levée du secret ou avertisse d'emblée les autorités afin de sauvegarder des intérêts prépondérants, tels que la sécurité publique.

En d'autres termes, le système actuel fonctionne.

\section{Lois romandes modifiées}

Les modifications législatives intervenues dans les cantons de Genève, Vaud et Valais ont un point commun: toutes prévoient que les médecins carcéraux engagés dans une relation thérapeutique avec un détenu sont soumis, dans certaines circonstances, à une obligation de signaler le détenu à l'autorité. Les circonstances envisagées sont essentiellement celles mentionnées dans la recommandation de la CLDJP: lorsque le médecin prend connaissance de faits importants concernant la dangerosité du détenu. Le but déclaré de cette réglementation est certes louable: renforcer la communication entre les acteurs du milieu carcéral et la 


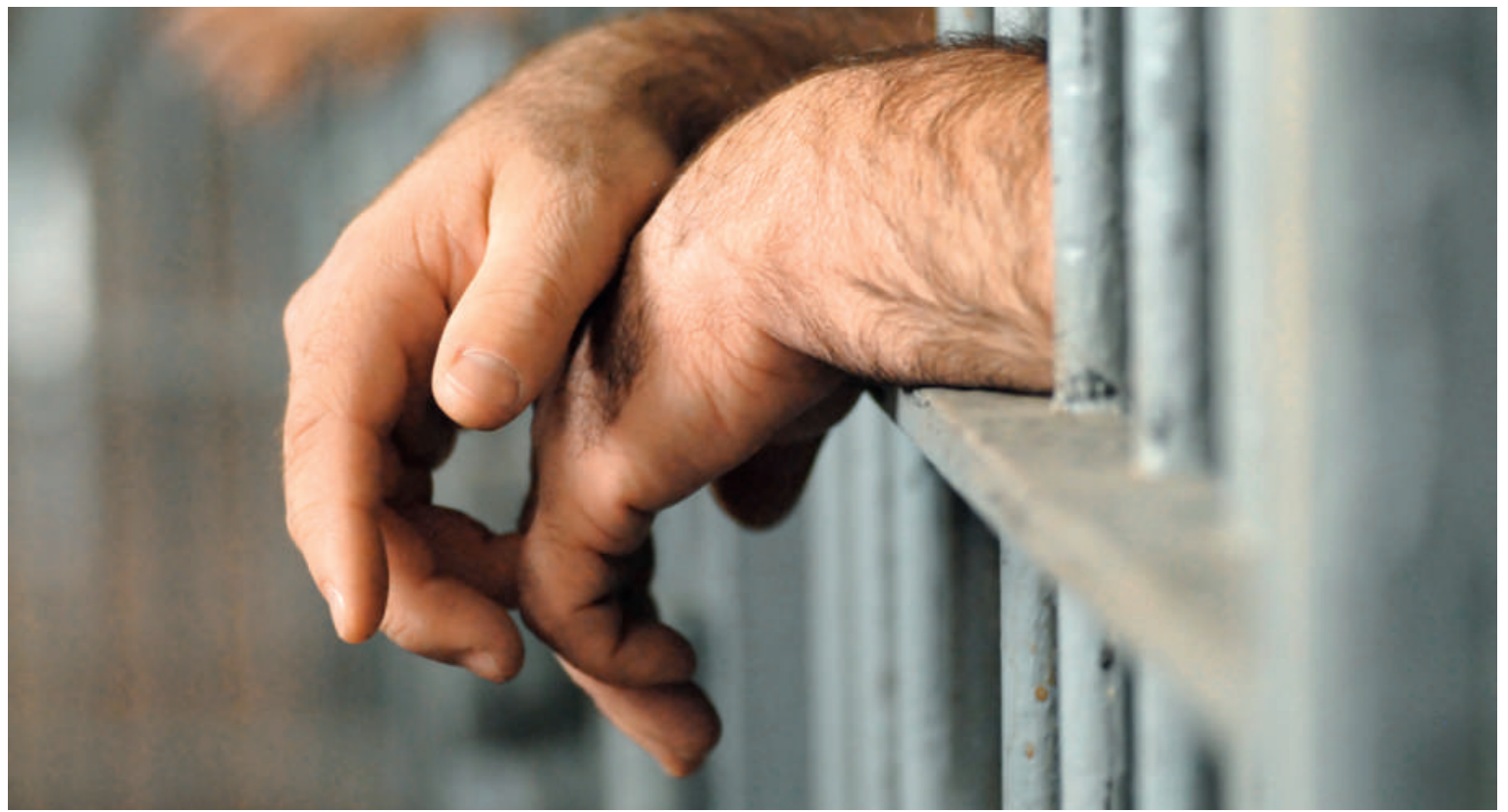

Les modifications législatives intervenues dans les cantons de Genève, Vaud et Valais ont un point commun: toutes prévoient que les médecins carcéraux engagés dans une relation thérapeutique avec un détenu sont soumis, dans certaines circonstances, à une obligation de signaler le détenu à l'autorité. Le médecin devrait alerter l'autorité de tout fait important en matière de dangerosité du détenu.

sécurité publique. Malheureusement, le choix de soumettre les médecins carcéraux à une obligation de dénoncer aura un effet inverse.

\section{Destruction de la relation de confiance}

Le médecin carcéral engagé dans une relation thérapeutique avec un détenu reste médecin à part entière («principe d'équivalence»), que cette relation découle de la seule volonté du détenu malade ou d'une mesure thérapeutique décidée par le juge. Le médecin carcéral est ainsi soumis au secret médical au même titre que le médecin traitant exerçant en milieu ouvert. Cette confidentialité est la garantie d'une relation de confiance, indispensable à une bonne communication et à un traitement efficace. Les lois modifiées détruisent cette relation de confiance, le médecin devenant un véritable auxiliaire de l'autorité. Il sera en effet impossible pour le détenu qui se confie au médecin de déterminer quelles informations resteront confidentielles et quelles informations seront transmises à l'autorité parce que considérées comme révélatrices de sa dangerosité.

\section{Danger pour la sécurité publique}

La destruction de la relation de confiance indispensable à la relation thérapeutique entraînera d'une part un traitement moins efficace, notamment sur le plan psychiatrique, et d'autre part la méfiance du détenu. Le détenu dont le traitement n'est pas optimal aura un risque de récidive plus élevé à sa sortie, et ne livrera plus certaines confidences qui jusqu'à présent pouvaient déterminer le médecin à alerter l'autorité. Un traitement moins efficace et un défaut de communication vont clairement à l'encontre de la sécurité publique.

\section{Mythe de l'expert permanent}

Les lois modifiées opèrent une grave confusion en faisant du médecin carcéral un "expert permanent», chargé en tout temps d'alerter l'autorité de tout fait important en matière de dangerosité. Or le médecin

\section{Le détenu dont le traitement n'est pas optimal} aura un risque de récidive plus élevé à sa sortie,

carcéral n'est ni formé, ni qualifié à l'évaluation de la dangerosité des détenus. Qu'il agisse dans le cadre du service médical de la prison ou dans le cadre d'une mesure thérapeutique ordonnée par le juge, il est avant tout un médecin traitant et non un expert en dangerosité. Le code pénal lui-même insiste sur la distinction entre médecin traitant et expert en dangerosité Lorsqu'il s'agit, chez une personne ayant commis un crime grave au sens de l'art. 64 al. $1 \mathrm{CP}^{2}$, de décider d'un allégement dans l'exécution d'une peine ou d'une mesure (congés, autorisation de travailler ou de loger à l'extérieur, libération conditionnelle, etc.) (art. 75a CP), de la levée d'une mesure (art. $62 \mathrm{~d}$ al. $1 \mathrm{CP}$ ) ou de l'insti- 
tution d'une mesure thérapeutique ou d'un internement (art. 56 al. 4 et $4^{\text {bis }} \mathrm{CP}$ ), une expertise doit être effectuée. Le code pénal précise: «L'expert et les représentants des milieux de la psychiatrie ne doivent ni avoir traité l'auteur ni s'être occupés de lui d'une quelconque manière» (art. $62 \mathrm{~d}$ al. $2 \mathrm{CP})^{3}$.

Les lois modifiées détruisent cette relation de confiance, le médecin devenant un véritable auxiliaire de l'autorité.

\section{Pourquoi seulement les professionnels de la santé?}

L'art. 321 CP consacré au secret professionnel est applicable non seulement aux professionnels de la santé mais également aux avocats et aux aumôniers, qui sont nombreux en milieu carcéral. Dans la mesure où les médecins carcéraux soumis à l'obligation de signaler ne sont ni formés, ni qualifiés à l'évaluation de la dangerosité des détenus, les informations sur la dangerosité qu'ils sont susceptibles de détenir ne sont pas plus spécifiques que celles que pourraient recueillir les avocats ou les aumôniers. Dès lors, il est permis de s'étonner que les lois modifiées ne visent que les professionnels de la santé en milieu carcéral et non les autres professions mentionnées à l'art. $321 \mathrm{CP}$. Il est sans doute aisé aux milieux politiques, dont les rangs comptent de nombreux avocats, de concevoir que les avocats ne pourraient plus exercer leur profession s'ils devaient être soumis à une obligation de signaler leurs clients. Il est regrettable que ce raisonnement leur échappe en ce qui concerne les professionnels de la santé.

\section{Transfert de responsabilité des autorités vers le médecin}

La plupart des détenus sont régulièrement en contact avec un médecin carcéral lors de leur privation de liberté. Soumis à une obligation de signaler, le médecin carcéral devient ainsi une cible naturelle pour le cas où le détenu - qui a bénéficié d'un allégement dans l'exécution au sens de l'art. 75a al. 2 CP, ou dont la mesure a été levée - commet une nouvelle infraction. On pourra en effet facilement reprocher a posteriori au médecin carcéral de ne pas avoir transmis aux autorités les informations relatives au détenu dont la dangerosité a désormais été démontrée. Ce transfert de responsabilité des autorités vers les professionnels de la santé résonne d'une manière toute particulière si l'on songe au drame qui a coûté la vie à Adeline, survenu alors que les autorités avaient omis d'effectuer une expertise pourtant prévue - de la dangerosité de l'assassin.

\section{Conclusion}

Les lois modifiées d'application du code pénal des cantons de Genève, Vaud et Valais constituent une «fausse bonne idée», contraire au principe fondamental du secret médical. Détruisant la relation de confiance indispensable à toute relation thérapeutique, cette régle-

\section{Contrairement à leur but déclaré, les lois modifiées vont à l'encontre de la sécurité publique.}

mentation va à l'encontre de son but déclaré de sécurité publique. A Genève, l'Association des Médecins (AMG) a lancé le 4 mars 2016 une initiative législative cantonale formulée. Celle-ci reprend l'essentiel du texte de la loi genevoise modifiée afin d'en conserver les objectifs louables tout en rétablissant le principe fondamental du secret médical. Le texte de l'initiative confirme ainsi le droit du médecin d'alerter l'autorité dans des situations qualifiées, mais écarte toute obligation de signaler à sa charge.

\section{Crédit photo:}

Willeecole | Dreamstime.com 